\title{
Primary localized conjunctival amyloidosis: A case report with a ten-year follow-up period
}

\author{
Juan Carlos Mesa-Gutiérrez \\ Tomás Martí Huguet \\ Noemí Barnils Garcia \\ Jorge Arruga Ginebreda \\ Department of Ophthalmology, \\ Hospital Universitari de Bellvitge, \\ L'Hospitalet de Llobregat, Barcelona, \\ Spain
}

Correspondence: Juan Carlos MesaGutiérrez

Department of Ophthalmology, Hospital Universitari de Bellvitge, Feixa Llarga s/n 08907, L'Hospitalet de Llobregat,

Barcelona, Spain

Tel +3493260 7705

Fax +3493260 798।

Email juancarlosmesa@lycos.co.uk

\begin{abstract}
A 57-year-old woman consulted our department about a plaque that had been present in her left eye for one year. Ophthalmological examination revealed a solid mass in the left conjunctival semilunar fold. An excitional biopsy was peformed, revealing an amyloid deposition. The patient was referred to the Internal Medicine Department for systemic study. All studies were negative for systemic amyloidosis. Throughout ten years of follow-up study, the patient has shown neither conjunctival recurrence nor any evidence of systemic amylodosis. Despite the fact that several authors have described ocular amyloidosis as an ophthalmologic manifestation of systemic amyloidosis, conjunctival location of amyloidosis is a very rare condition. Amyloid characterization in ocular structures should not be considered a final diagnosis since involvement of systemic underlying diseases must be ruled out. We report a case of localized conjunctival amyloidosis with a ten-year follow-up period that excluded ocular or systemic diseases.
\end{abstract}

Keywords: conjuctival amyloidosis, ocular amyloidosis, hyaline protein

\section{Introduction}

Amyloidosis is defined as the deposition of an eosinophilic hyaline protein in the tissues. The classification includes four types (Westermark et al 2002):

1. Primary localized amyloidosis,

2. Primary systemic amyloidosis,

3. Secondary localized amyloidosis, and

4. Secondary systemic amylodosis.

We present the case of a patient with primary localized conjunctival amyloidosis who showed no evidence of ocular or systemic manifestation by the amyloid after a ten-year follow-up period.

\section{Case report}

A systemically healthy 57-year-old female consulted our department after becoming concerned about a lightly colored plaque that had been present in her left eye for one year. The ophthalmologic examination revealed a visual acuity of $20 / 20$. The anterior segment examination revealed a smooth, solid mass measuring $5 \times 8 \mathrm{~mm}$ located in the stroma of the left conjunctival semilunar fold. The intraocular pressure measurement was $14 \mathrm{mmHg}$ for the left eye. Fundus examination was normal and the right eye was unaffected (Figure 1).

As a conjunctival neoplasm was suspected, a conjunctival biopsy was performed. The lesion was excised in its entirety. The specimen revealed a deposit of submucosal amorphous eosinophilic material. Congo red stain demonstrated the characteristic salmon pink color of these deposits. Under polarized light, the Congo red-stained material revealed a green birefringence characteristic of amyloid deposition. This reaction was eradicated by pretreatment with $\mathrm{KM}_{\mathrm{n}} \mathrm{O}_{4}$, consistent with the AA (amyloidassociated) subtype. There was no associated lymphocytic infiltration (Figure 2). 


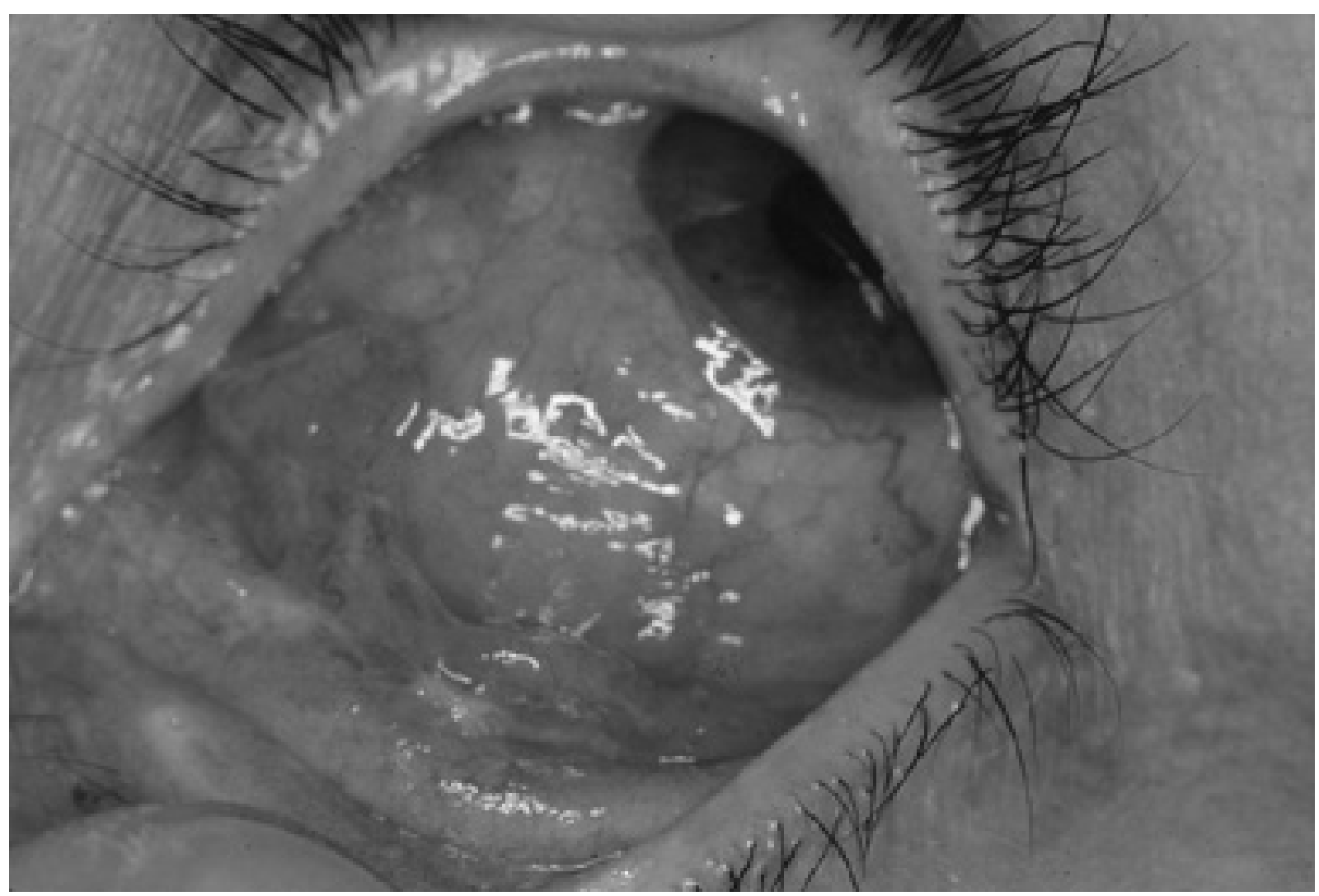

Figure I Biomicroscopy (Left eye).

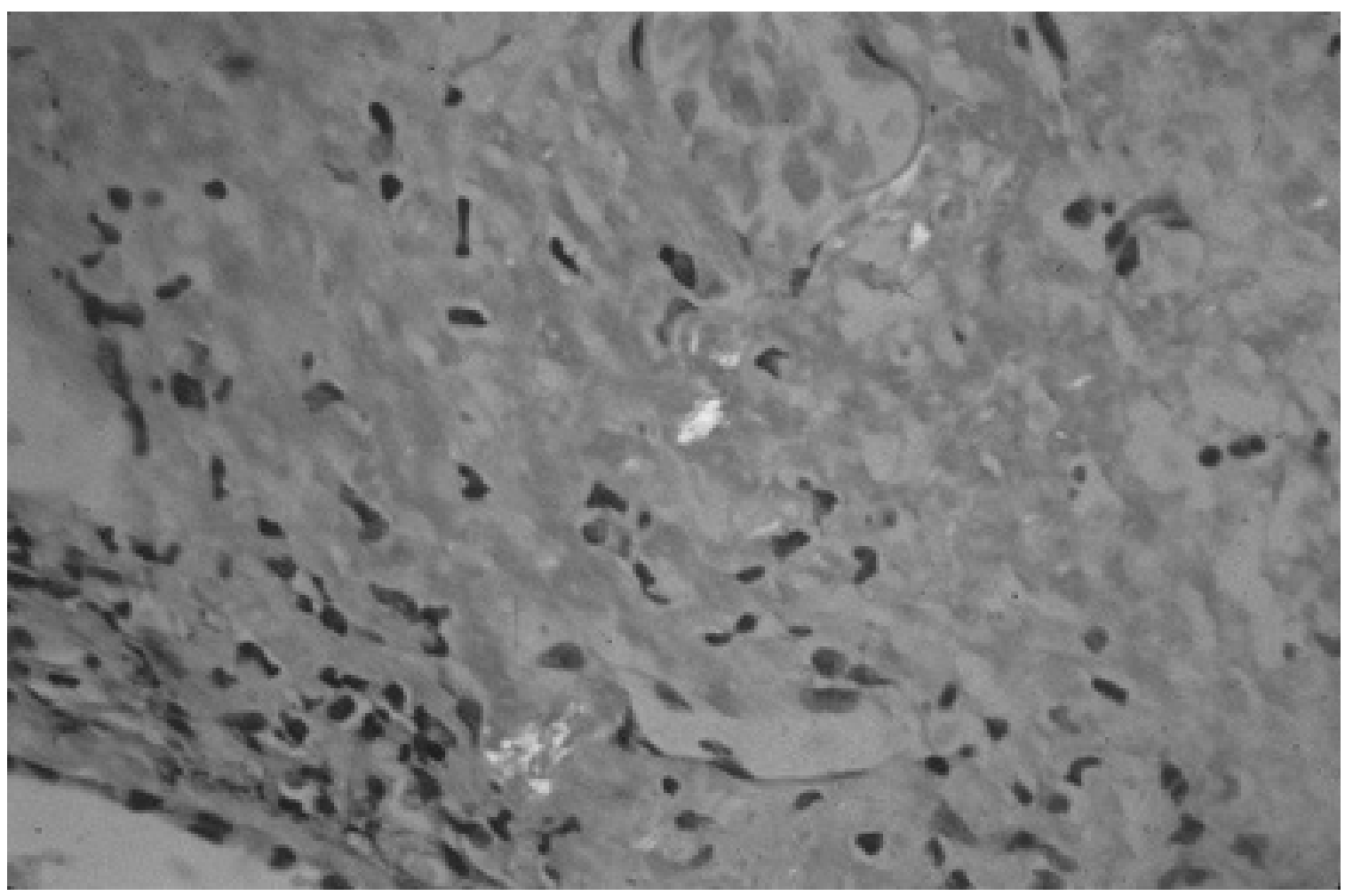

Figure 2 Amyloid characterization: Congo red-stained material revealing green birefringence diagnostic of amyloid deposition. 
The patient underwent further systemic studies. The work-up included a complete blood count, urinalysis, and urine protein electrophoresis that were within normal limits. Whereas a simple needle biopsy of clinically uninvolved abdominal subcutaneous fat is positive in $95 \%$ of primary systemic amyloidosis cases, biopsies were performed in this case and the results were negative for Congo red staining (Lee et al 2000; Westermark et al 2002).

After a ten-year follow-up period, the patient has not shown any recurrence. There has been no evidence of ocular or systemic diseases, and no clinical or analytical data supporting a diagnosis of systemic amyloidosis. The patient remains in good health and is currently asymptomatic.

\section{Discussion}

Primary ocular amyloidosis of the eye, especially conjunctival amyloidosis, is a rare clinical occurrence that is mainly diagnosed by histopathological examination and must be considered in the differential diagnosis of conjunctival neoplasms (Moorman and McDonald 1997; Lee et al 2000).

One of the characteristic features of an amyloidosis is its tendency to bleed. That was not a feature in our case (Lee et al 2000; Pirouzmand et al 2002; Westermark et al 2002).

Diagnosis at an early stage is difficult. Yellowish deposits are indicative of amyloidosis, but are not obvious in the early stages. The salmon pink color of the conjunctiva suggests a lymphoma or even leukaemia. Histopathologic examination is necessary in order to determine the final diagnosis, as this is the only way to demonstrate amyloid material in tissue (Rosenblatt et al 1986; Pirouzmand et al 2002).

The mere recognition of an amyloid in an ocular structure should not be considered a complete diagnosis. The possibility of amyloidosis in extraocular sites associated with underlying diseases or immunoglobulin abnormalities must be considered (Lee et al 2000; Pirouzmand et al 2002; Westermark et al 2002). This possibility has been ruled out in our case after ten years of periodic investigation for systemic diseases.

To our knowledge, this is the first case of conjunctival amyiloidosis with ten years of documented work-up excluding ocular or systemic diseases.

\section{Discolsure}

The authors report no conflicts of interest in this work.

\section{References}

Lee HM, Naor J, DeAngelis D, Rootman D. 2000. Primary localizad conjunctival amyloidosis presentig with recurrente of subconjunctival hemorrhage. Am J Ophthalmol, 129:244-5.

Moorman CM, McDonald B. 1997. Primary (localized non-familial) conjunctival amyloidosis: three case reports. Eye, 11:603-6.

Pirouzmand F, Hurwitz J, Howarth D. 2002. Primary localised bilateral conjunctival amyloidosis and epiphora. Orbit, 21:139-44.

Rosenblatt M, Spitz GF, Friedman AH, et al. 1986. Localized conjunctival amyloidose: case reports and review of literature. Ophthalmologica, 192:238-45.

Westermark P, Benson MD, Buxbaum JN, et al. 2002. Amyloid fibril protein nomenclature - 2002. Amyloid, 9:197-200. 